\title{
Regulation of major vault protein expression by upstream stimulating factor 1 in SW620 human colon cancer cells
}

\author{
RYUJI IKEDA $^{1}$, YUKIHIKO NISHIZAWA ${ }^{1}$, YUSUKE TAJITSU ${ }^{1}$, KENTARO MINAMI ${ }^{1}$, \\ HIRONORI MATAKI ${ }^{1}$, SHOGO MASUDA $^{1}$, TATSUHIKO FURUKAWA ${ }^{2}$, SHIN-ICHI AKIYAMA ${ }^{3}$, \\ KATSUSHI YAMADA $^{4}$ and YASUO TAKEDA ${ }^{1}$
}

\begin{abstract}
Departments of ${ }^{1}$ Clinical Pharmacy and Pharmacology, ${ }^{2}$ Molecular Oncology, Graduate School of Medical and Dental Sciences, Kagoshima University, Kagoshima 890-8520; ${ }^{3}$ Department of Respiratory Medicine and Rheumatology, Institute of Health Biosciences, the University of Tokushima Graduate School, Tokushima 770-8503; ${ }^{4}$ Department of Clinical Pharmacology, Faculty of Pharmaceutical Sciences, Nagasaki International University, Nagasaki 859-3298, Japan
\end{abstract}

Received June 28, 2013; Accepted August 26, 2013

DOI: 10.3892/or.2013.2818

\begin{abstract}
Major vault protein (MVP) is the main constituent of the vault ribonucleoprotein particle and is identical to lung resistance-related protein (LRP). Although MVP is also expressed in several types of normal tissues, little is known about its physiological role. In the present study, we identified the crucial MVP promoter elements that regulate MVP expression. An examination of tissue expression profiles revealed that MVP was expressed in the heart, placenta, lung, liver, kidney and pancreas. Elements of the MVP promoter contain binding sites for transcription factors, STAT, p53, Sp1, E-box, GATA, MyoD and Y-box. By deletion analysis, a conserved proximal E-box binding site was demonstrated to be important for human $M V P$ promoter transactivation. Introduction of siRNA against upstream stimulating factor (USF) 1 , which is known to bind the E-box binding site, decreased the expression of MVP in SW620 and ACHN cells. Using a chromatin immunoprecipitation (ChIP) assay, USF1 bound the MVP promoter in SW620 cells. These findings suggest that USF1 binding to an E-box element may be critical for basal MVP promoter activation. The results of the present study are useful in understanding the molecular mechanisms regulating MVP gene expression, and may aid in elucidating the physiological functions of MVP.
\end{abstract}

\section{Introduction}

Vault particles are composed of multiple copies of three proteins, a major vault protein (MVP), also known as lung

Correspondence to: Professor Yasuo Takeda, Department of Clinical Pharmacy and Pharmacology, Graduate School of Medical and Dental Sciences, Kagoshima University, 8-35-1 Sakuragaoka, Kagoshima 890-8520, Japan

E-mail: takeda@m.kufm.kagoshima-u.ac.jp

Key words: major vault protein, upstream stimulating factor 1 resistance-related protein (LRP), a telomerase-associated protein, and a vault-poly(ADP-ribose) polymerase, as well as untranslated RNA molecules (1-3). The distribution of MVP in normal human tissues is similar to other drug resistancerelated proteins. MVP is expressed in tissues chronically exposed to xenobiotics. MVP expression is high in certain multidrug-resistant tumors (4) and increased MVP expression represents an early event in colorectal carcinogenesis (5). We previously reported that the expression of the MVP protein, $M V P$ mRNA and the promoter activity of $M V P$ were increased by osmostress (7). The downregulation of $M V P$ expression by MVP interfering RNA (RNAi) in SW620 cells increased the sensitivity of cells to hyperosmotic stress. These results suggest that the exposure of cells to hyperosmotic stress may induce MVP, which may play an important role in protecting cells from the adverse effects of osmotic stress. Although the expression of MVP was also associated with an adverse clinical outcome and shorter overall survival (6), little is known about the regulation of $M V P$ expression. The aim of the present study was to investigate the molecular basis for $M V P$ gene expression in human SW620 colon cancer cells.

\section{Materials and methods}

Materials. SW620, a human colon carcinoma cell line, was provided by Dr A.T. Fojo (National Cancer Institute, Bethesda, MD, USA). RPMI-1640 medium was purchased from Nissui Seiyaku Co. (Tokyo, Japan), and fetal calf serum (FCS) was from JRH Biosciences (Lenexa, KS, USA). A rabbit anti-ERK polyclonal antibody was obtained from Santa Cruz Biotechnology (Santa Cruz, CA, USA). A rabbit antiMVP polyclonal antibody was prepared using a glutathione S-transferase (GST)-MVP (aa 694-794) fusion protein as an antigen (4). Multiple tissue cDNA panels were from Clontech Laboratories Inc. (Mountain View, CA, USA). Anti- $\alpha$-tubulin antibody was from Calbiochem (Darmstadt, Germany).

Cell culture. SW620 and ACHN cells, a human renal adenocarcinoma cell line, were grown in RPMI-1640 containing $10 \%$ FCS, $2 \mathrm{mM}$ glutamine and $100 \mathrm{U} / \mathrm{ml}$ of penicillin at 
$37^{\circ} \mathrm{C}$ in a $5 \% \mathrm{CO}_{2}$ humidified atmosphere. Cell cultures were maintained in exponential growth by media replacement every 2-3 days. Cells were provided with fresh media the day before use for experimentation.

Fluorescence and laser scanning confocal microscopy (LSCM). Imaging was conducted using a confocal laser scanning microscope (FV500; Olympus Corp., Tokyo, Japan). SW620 cells $\left(1 \times 10^{6}\right)$ were incubated for $24 \mathrm{~h}$ at $37^{\circ} \mathrm{C}$. After being carefully washed twice with PBS, SW620 cells were fixed with $3 \%$ formaldehyde for $30 \mathrm{~min}$ at room temperature. They were then washed twice with PBS and permeabilized with $100 \%$ methanol for $20 \mathrm{~min}$ at room temperature. After blocking for 30 min with $3 \%$ skimmed milk in PBS, cells were incubated for $1 \mathrm{~h}$ with a polyclonal antibody against MVP diluted in PBS containing 3\% skimmed milk. After washing three times in PBS, cells were incubated for 20 min with FITC-conjugated goat anti-rabbit IgG diluted 1:100 in PBS containing 3\% skimmed milk. Cells were then washed three times in PBS and samples were examined by confocal microscopy. Fluorescence was imaged at an excitation wavelength $\left(\lambda_{\mathrm{ex}}\right)=488$ and with an emission bandpass filter set for FITC.

Immunoblot analysis. Samples were subjected to 6 or $12.5 \%$ sodium dodecyl sulfate polyacrylamide gel electrophoresis (SDS-PAGE) according to the method of Laemmli. Gel proteins were electrophoretically transferred onto polyvinylidene difluoride membranes (Immobilon-P transfer membrane; Millipore, Bedford, MA, USA) using the Bio-Rad Trans-Blot SD apparatus. The membrane was treated with a buffer [350 mM NaCl, 10 mM Tris- $\mathrm{HCl}$ (pH 8.0), 0.05\% Tween-20] containing $3 \%$ skimmed milk for $1 \mathrm{~h}$ and incubated with the indicated antibody $(1: 1,000)$ in the buffer containing $3 \%$ skimmed milk for $1 \mathrm{~h}$. Following four washes with the buffer (10 min each), the membrane was incubated with a peroxidaseconjugated horse anti-rabbit $\operatorname{IgG}$ diluted 1:1,000 in the buffer containing $3 \%$ skimmed milk for $1 \mathrm{~h}$. The membrane was washed with buffer, and developed using the enhanced chemiluminescence western blotting detection system (Amersham Pharmacia, Buckinghamshire, UK).

Dual-luciferase reporter assay. The human MVP promoter ranging from -407 and -78 to +66 was amplified from the genomic DNA of SW620 cells as previously described (22). The PCR product was cloned into the pT7 Blue-2 vector (Merck Biosciences, San Diego, CA, USA) and sequenced. Following digestion with KpnI and SacI, the $M V P$ promoter fragment was gel-purified and ligated into the KpnI and SacI-linearized pGL3-Basic vector (Promega, Madison, WI, USA). The resulting constructs were designated pMVP407 and pMVP78 (22). SW620 cells were grown in RPMI-1640 medium containing 10\% FCS, 2 mM glutamine, and $100 \mathrm{U} / \mathrm{ml}$ of penicillin at $37^{\circ} \mathrm{C}$ in a $5 \% \mathrm{CO}_{2}$ humidified atmosphere. Cells $\left(3.0 \times 10^{5} /\right.$ well in 6 -well plates) were transfected with $1 \mu \mathrm{g}$ of luciferase reporter plasmid (pGL3, Promega) or $100 \mathrm{ng}$ of control plasmid (pRL-TK; Promega). Following incubation for $24 \mathrm{~h}$ with the transfected cells, the luciferase assay was performed using the Dual-Luciferase Reporter Assay System following the manufacturer's protocol (Promega). Luminescence assays were performed using a luminometer
(TD-20/20 Luminometer; Turner Designs, Sunnyvale, CA, USA). All experiments were performed in triplicate and the results were normalized to pRL-TK activity.

RNA interference. Small interfering RNA (siRNA) duplexes were synthesized using the Silencer ${ }^{\circledR}$ siRNA construction kit (Ambion Inc.). A target site within the gene was chosen from the mRNA sequence of USF1. Following selection, the target site was searched with the National Center for Biotechnology Information Blast to confirm its specificity. The siRNA used in the present study consisted of a 21-nucleotide sense strand and a 21-nucleotide antisense strand with a two-nucleotide overhang at the end. Sequences were as follows: USF1-siRNA sense, 5'-GGAAGGUGCAGUGGCUACUGG-3', starting at nucleotide 54 from the AUG start codon of the human USF1 coding sequence; USF1-siRNA antisense, 5'-GGCCAGUAG CCACTGCACCUU-3'; siRNA was transfected to cells using Lipofectamine $2000^{\mathrm{TM}}$ according to the manufacturer's instructions (Invitrogen, Carlsbad, CA, USA).

RT-PCR method. Total cellular RNA was extracted using TRIzol reagent according to the manufacturer's instructions (Invitrogen). RT-PCR was performed with the SuperScript One-Step RT-PCR system and gene-specific primers according to the manufacturer's instructions (Invitrogen). Reaction mixtures containing total RNA (500 ng of each), $0.2 \mu \mathrm{M}$ of each primer, and an enzyme mixture composed of SuperScript II RT, Platinum Taq DNA polymerase, were maintained at $50^{\circ} \mathrm{C}$ for $20 \mathrm{~min}$, then at $94^{\circ} \mathrm{C}$ for $2 \mathrm{~min}$, and PCR was performed as follows: 30 cycles at $94^{\circ} \mathrm{C}$ for $15 \mathrm{sec}, 55^{\circ} \mathrm{C}$ for $30 \mathrm{sec}$, and $70^{\circ} \mathrm{C}$ for $30 \mathrm{sec}$. Primers for RT-PCRs were designed based on human sequences in GenBank. These sequences used the following primers: $M V P$ (GenBank accession no. NM_017458), 5'-CAGGATGTGTATGTGCTGT CGG-3' and 5'-GCTGGAGGCTCTTAGCTGTGTC-3'; USF1 (GenBank accession no. NM_ 007122), 5'-ATGAAGGGG CAGCAGAAAACA-3' and 5'-TTAGTTGCTGTCATTCT TGA-3' and GAPDH (GenBank accession no. NM_002046), 5'-AGAACATCATCCCTGCCTCTACTGG-3' and 5'-AAAG GTGGAGGAGTGGGTGTCGCTG-3'.

Real-time reverse-transcription PCR quantification. Total cellular RNA was extracted using TRIzol reagent according to the manufacturer's instructions (Invitrogen). One microgram of RNA was reverse transcribed using a first-strand cDNA synthesis kit (ReverTra Ace ${ }^{\circledR}$; Toyobo, Osaka, Japan). Human $M V P$ and GAPDH gene expression levels were assayed by real-time reverse PCR (PRISM 7900HT; Applied Biosystems, Foster City, CA, USA) according to the technical specifications. Human GAPDH was used for normalization. The quantification of target gene expression was obtained with the comparative cycle threshold method according to the instructions of the manufacturer.

Chromatin immunoprecipitation assay. Cells were fixed with $1 \%$ formaldehyde for $10 \mathrm{~min}$ at $37^{\circ} \mathrm{C}$ to cross-link protein to DNA. A chromatin immunoprecipitation (ChIP) assay was carried out using a ChIP assay kit (Upstate Biotechnology) according to the manufacturer's instructions. The soluble DNA fraction was mixed with an anti-USF-1 antibody (Santa Cruz 


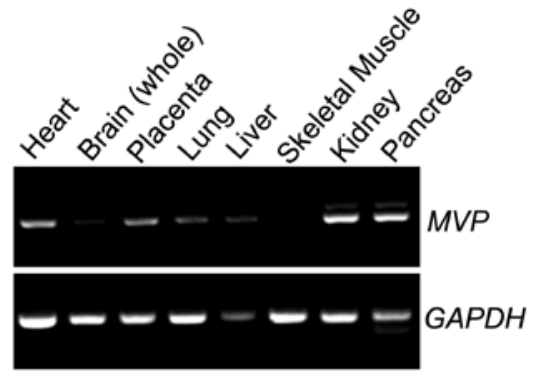

Figure 1. Expression of the human $M V P$ gene. Expression levels of $M V P$ mRNA in various human tissues. GAPDH mRNA was also detected as a control.

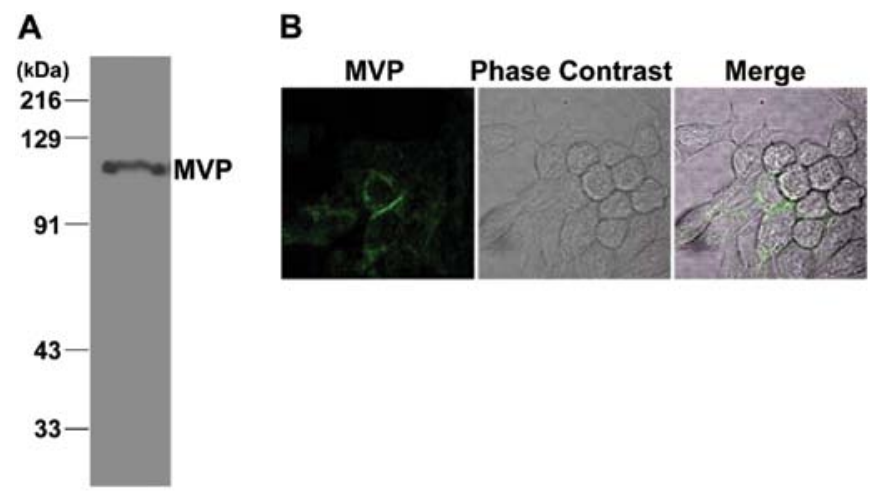

Figure 2. Expression levels of the MVP protein in SW620 cells. (A) Cells were examined by immunoblot analysis. Total lysates $(100 \mu \mathrm{g})$ from cells were separated by SDS-PAGE and immunoblotted with an anti-MVP polyclonal antibody. (B) Intercellular localization of MVP in SW620 cells. Cells were fixed and immunostained for MVP.

Biotechnology) or non-immunized mouse IgG (Santa Cruz Biotechnology) and the precipitated DNA was amplified with primers for the $M V P$ promoter, 5'-GCCAGCTGGCTCCAAG GTAG-3' (sense) and 5'-GGCAGGGCAAGGCAGGCCAA-3' (antisense).

A

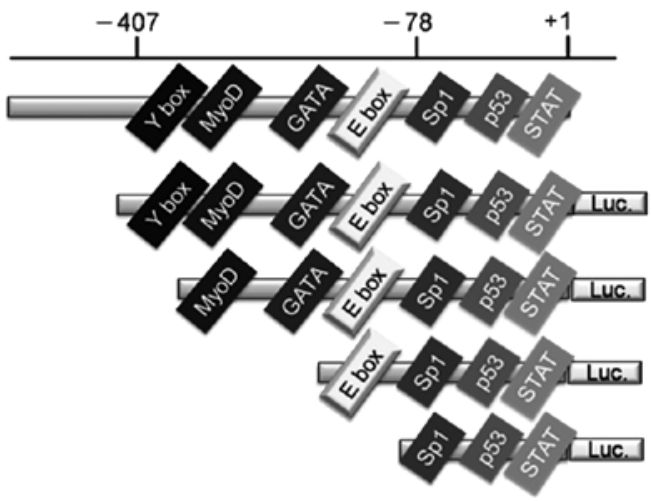

\section{Results}

Tissue expression profiles of MVP $m R N A$. At the start of the present study, we examined the tissue expression profile of MVP mRNA by performing PCR. MVP was found to be expressed in the heart, placenta, lung, liver, kidney and pancreas in normal tissues (Fig. 1).

Expression of MVP in cancer cell lines and intracellular localization of MVP. To examine if the MVP protein was expressed in cancer cell lines, we cultured human colon adenocarcinoma SW620 cells. As shown in Fig. 2A, the MVP protein was expressed in SW620 cells. It is known that MVP can shuttle between the nucleus and the cytoplasm. We examined the localization of MVP in SW620 cells by immunofluorescence. MVP was mainly localized in the cytoplasm of SW620 cells (Fig. 2B).

Identification of MVP cis-elements responsible for the transcription activity of the 5'-flanking region of the MVP gene. To confirm basal MVP gene transcription, we cloned human $M V P$ promoter fragments and inserted them into a luciferase reporter vector. One construct, pMVP407, contained all the known conserved promoter elements, Y-box, Myo D, GATA, E-box, Sp1, p53 and STAT of the $M V P$ promoter (22). Following transfection of the individual plasmids into SW620 cells, the cells and $M V P$ promoter activity was monitored by a luciferase assay (Fig. 3). Luciferase activity in pMVP407 and pMVP263-transfected cells was 200 -fold higher than that of cells transfected with the control luciferase vector. The level of luciferase activity was gradually reduced as the fragment became shorter. Basal luciferase activity in pMVP78-transfected cells was significantly lower than that in pMVP102-transfected cells. The construct pMVP78 lacks the E-box binding site of the construct pMVP102. These findings suggest that the E-box-binding motif may play a crucial role in maintaining basal MVP promoter activity.

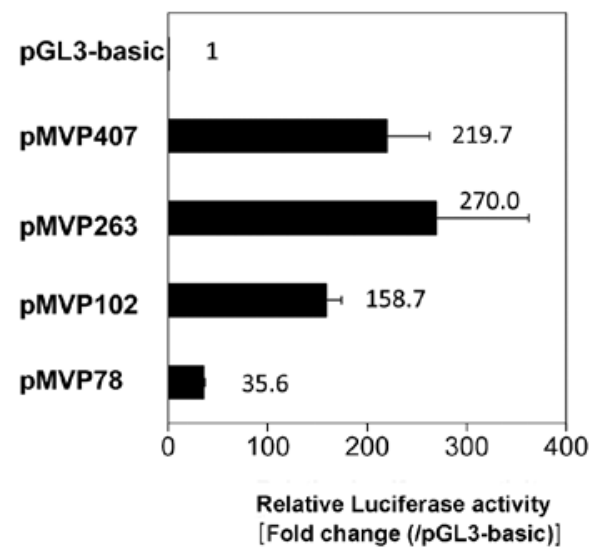

Figure 3. MVP promoter-reporter gene expression in SW620 cells. (A) Outline of the MVP promoter construct used. The top line indicates the promoter region (-407 to 1) upstream of the MVP transcription start site. The positions of all the known conserved promoter elements, Y-box, MyoD, GATA, E-box, Sp1, p53 and STAT, are indicated below. The construct was linked to a luciferase reporter gene (luc). (B) SW620 cells were transiently transfected with pMVP (1 $\mu \mathrm{g})$ and pRL-TK (100 ng) reporter gene constructs using the Lipofectamine reagent. Following transfection, cells were incubated for $24 \mathrm{~h}$ and then assayed for luciferase activity. Experiments were performed in triplicate and the results were normalized to pRL-TK activity. 

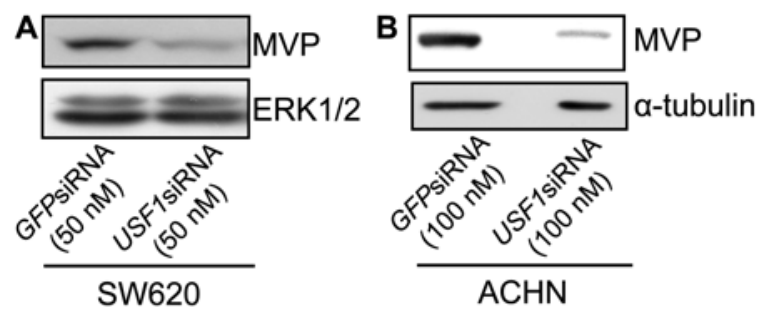

Figure 4. Effect of the siRNA-mediated gene silencing of USF1 on the expression of MVP in (A) SW620 cells and (B) ACHN cells. SW620 cells transfected with siRNA targeting USF1 or GFP were cultured for $24 \mathrm{~h}$, and cell lysates were prepared from these cells. The expression of MVP was detected by immunoblotting using an anti-MVP antibody or an anti-ERK antibody.
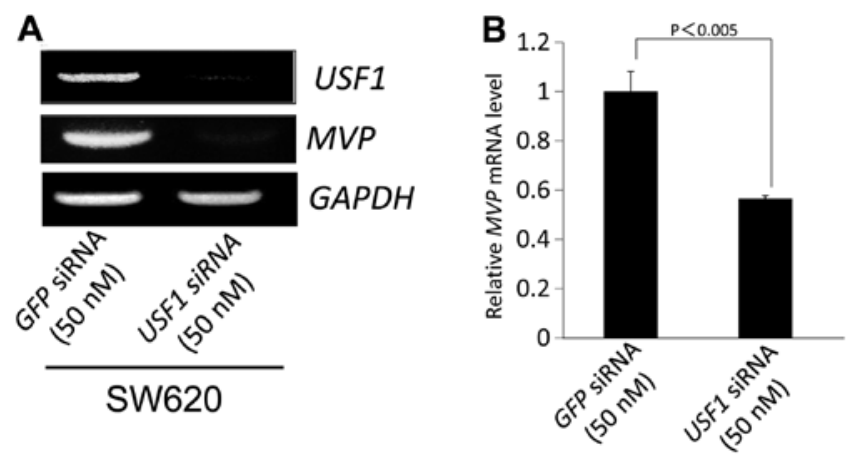

Figure 5. Effect of USF-1 knockdown on the expression of $M V P$ mRNA in SW620 cells. (A) SW620 cells were transfected with USF1 siRNA for $24 \mathrm{~h}$, and RT-PCRs were performed with specific primers for $U S F 1, M V P$ and $G A P D H$. (B) $M V P$ mRNA levels were measured using real-time RT-PCR. The GAPDH gene was used to normalize the value of $M V P$ mRNA.

The effect of USF1 on the expression of MVP in SW620 cells. USF1 and USF2 are members of the eukaryotic evolutionary conserved Basic-Helix-Loop-Helix Leucine Zipper transcription factor. They interact with high affinity to cognate E-box regulatory elements and are ubiquitously expressed. To examine whether the expression of USF1 regulates the expression of MVP, SW620 cells transfected with siRNA duplexes targeting $U S F 1$ or green fluorescent protein $(G F P)$ as a control were cultured. As shown in Fig. 4A, the introduction of siRNA against $U S F 1$ resulted in a downregulation in MVP expression in SW620 cells. To examine the effects in ACHN cells, ACHN cells were transfected with siRNA duplexes targeting $U S F 1$ or green fluorescent protein $(G F P)$. The introduction of siRNA against USF1 also resulted in a downregulation in MVP expression in ACHN cells. These results indicate that the expression of $M V P$ is affected by the expression of USF1 in SW620 cells.

The effect of USF1 on MVP mRNA levels in SW620 cells. To determine if $M V P$ mRNA expression was altered by the introduction of siRNA against $U S F 1$, we examined the siRNA against USF1 on the expression levels of MVP in SW620 cells by RT-PCR and real-time RT-PCR. RT-PCR and real-time RT-PCR analyses confirmed that the introduction of siRNA against $U S F 1$ in SW620 cells downregulated the expression of USF1 and consequently suppressed MVP mRNA levels in SW620 cells (Fig. 5A and B). These results suggest that USF1 modulates the expression of MVP.

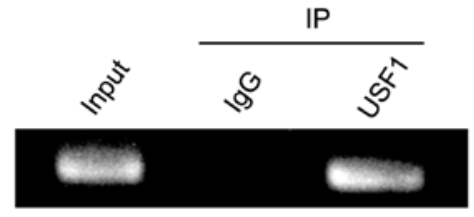

Figure 6. Binding of USF1 to the MVP promoter in SW620 cells. SW620 cells were cultured for $24 \mathrm{~h}$, and cells were fixed with formaldehyde to form a DNA-protein complex and subjected to a ChIP assay. PCR for the core promoter region of the $M V P$ gene was performed using DNA extracted from the DNA-protein complex immunoprecipitated using an anti-USF1 antibody or non-immune IgG.

Binding of USF1 to the MVP promoter in SW620 cells. To test for in vivo USF1 binding to the $M V P$ promoter, we performed a ChIP assay using the antibody against USF1. The chromatin immunoprecipitated by the antibody was then amplified by PCR with primers specific for MVP. As shown in Fig. 6, USF1 bound the MVP promoter in SW620 cells.

\section{Discussion}

The high evolutionary conservation and wide species distribution of vaults suggest an important role of vaults in cellular function. The human vault particle is a 13-MDa ribonucleoprotein complex comprised of the $\sim 100-\mathrm{kDa}$ major vault protein (MVP), 193-kDa vault-associated poly(ADP-ribose) polymerase (vPARP), and the $280-\mathrm{kDa}$ vault protein that has been demonstrated to be identical to a component of telomerase, TEP1, and four small untranslated vault RNAs (vRNAs) (1-3). MVP is expressed in the kidney, adrenal glands, heart, lung, muscle, thyroid, prostate, liver, pancreas, placenta, bone marrow and testis (8) (Fig. 1). These findings suggest that MVP plays distinct physiological roles in these organs. However, the physiological role of vaults remains unknown.

The MVP promoter contains conserved promoter elements, Y-box, Myo D, GATA, E-box, Sp1, p53 and STAT of the MVP promoter (9). YB-1 is a transcription factor that binds to the Y-box and responds to environmental stresses such as anticancer agents, UV light and hyperthermia (10-12). Treatment with IFN- $\gamma$ resulted in a significant upregulation of MVP promoter activity as well as mRNA and protein levels. The activation of MVP expression by IFN- $\gamma$ involved transcriptional upregulation through the JAK/STAT pathway based on the interaction of STAT1 within the proximal MVP promoter (13). Sp-transcription factors are essential for the basal MVP promoter via binding to the GC-box element with the $M V P$ promoter sequence, and stimulation of the MVP promoter was increased by HDAC inhibitors (14). As shown in Fig. 3B, deletion of the region from -102 to -79 , which contained the E-box element, resulted in a decrease in the level of transcriptional activity by $22.4 \%$ in SW620 cells. This result suggests that the E-box-binding motif may play a crucial role in maintaining basal MVP promoter activity in SW620 cells.

USF is a ubiquitous transcription factor in mammalian cells. Two different genes, defined as USF1 and USF2, have been isolated from HeLa cells, and these genes encode 43 and 44-kDa polypeptides, respectively (15-18). These two peptides have identical DNA-binding specificities and transcriptional activities (15-18). Although USF1 and USF2 polypeptides are 
highly divergent in their $\mathrm{N}$-terminal sequences, they share a highly conserved C-terminal basic/helix-loop-helix/leucine zipper (B-HLH-LZ) sequence that is important for the homoand heterodimerization and DNA-binding of USF (18). Proteins of the B-HLHLZ gene family bind to the DNA sequences of the general type CANNTG, which is referred to as the E-box $(19,20)$. In mammalian cells, two ubiquitously expressed genes, USF1 and USF2, have been characterized and have pleiotropic effects in cells and tissues $(18,21)$. The patterns of USF 1 and MVP genes were expressed in similar tissues; therefore, we investigated the effect of USF1 on the expression of MVP in SW620 cells (Fig. 1) (18). The introduction of siRNA against USF1 decreased the expression of MVP in SW620 cells (Fig. 4). These results indicated that the expression of MVP was regulated by USF1 in SW620 cells.

Our findings showed that a conserved proximal E-box binding site is important for basal human $M V P$ promoter transactivation by deletion analysis of the $M V P$ promoter assay and the introduction of siRNA against USF1 in SW620 cells also decreased the expression of MVP. These results suggest that USF1 binding to the E-box element may be critical for basal MVP promoter activation. However, further study is required to confirm whether USF1 and other transcription factors regulate the tissue-specific MVP expression involved in cell development and differentiation and malignant transformation.

\section{References}

1. Scheffer GL, Wijngaard PL, Flens MJ, Izquierdo MA, Slovak ML, Pinedo HM, Meijer CJ, Clevers HC and Scheper RJ: The drug resistance-related protein LRP is the human major vault protein. Nat Med 1: 578-582, 1995.

2. Scheper RJ, Broxterman HJ, Scheffer GL, Kaaijk P, Dalton WS, van Heijningen TH, van Kalken CK, Slovak ML, de Vries EG, van der Valk P, Meijer CJ and Pinedo HM: Overexpression of a M(r) 110,000 vesicular protein in non-P-glycoprotein-mediated multidrug resistance. Cancer Res 53: 1475-1479, 1993.

3. Scheffer GL, Schroeijers AB, Izquierdo MA, Wiemer EA and Scheper RJ: Lung resistance-related protein/major vault protein and vaults in multidrug-resistant cancer. Curr Opin Oncol 12 $550-556,2000$

4. Kitazono M, Sumizawa T, Takebayashi Y, Chen ZS, Furukawa T, Nagayama S, Tani A, Takao S, Aikou T and Akiyama S: Multidrug resistance and the lung resistance-related protein in human colon carcinoma SW-620 cells. J Natl Cancer Inst 91: $1647-1653,1999$

5. Pohl G, Suchomel RW, Stranzl T, Depisch D, Stiglbauer W, Filipits $\mathrm{M}$ and Pirker R: Expression of the lung resistance protein in primary colorectal carcinomas. Anticancer Res 21: 201-204, 2001.

6. Meijer GA, Schroeijers AB, Flens MJ, Meuwissen SG, van der Valk P, Baak JP and Scheper RJ: Increased expression of multidrug resistance related proteins Pgp, MRP1, and LRP/MVP occurs early in colorectal carcinogenesis. J Clin Pathol 52: 450-454, 1999.
7. Ikeda R, Iwashita K, Sumizawa T, Beppu S, Tabata S, Tajitsu Y, Shimamoto Y, Yoshida K, Furukawa T, Che XF, Yamaguchi T, Ushiyama M, Miyawaki A, Takeda Y, Yamamoto M, Zhao HY, Shibayama Y, Yamada K and Akiyama S: Hyperosmotic stress up-regulates the expression of major vault protein in SW620 human colon cancer cells. Exp Cell Res 314: 3017-3026, 2008.

8. Sugawara I, Akiyama S, Scheper RJ and Itoyama S: Lung resistance protein (LRP) expression in human normal tissues in comparison with that of MDR1 and MRP. Cancer Lett 112: 23-31, 1997.

9. Lange C, Walther W, Schwabe $\mathrm{H}$ and Stein U: Cloning and initial analysis of the human multidrug resistance-related MVP/LRP gene promoter. Biochem Biophys Res Commun 278: 125-133, 2000.

10. Asakuno K, Kohno K, Uchiumi T, Kubo T, Sato S, Isono M and Kuwano M: Involvement of a DNA binding protein, MDR-NF1/YB-1, in human MDR1 gene expression by actinomycin D. Biochem Biophys Res Commun 199: 1428-1435, 1994.

11. Ohga T, Uchiumi T, Makino Y, Koike K, Wada M, Kuwano M and Kohno K: Direct involvement of the Y-box binding protein YB-1 in genotoxic stress-induced activation of the human multidrug resistance 1 gene. J Biol Chem 273: 5997-6000, 1998.

12. Stein U, Jürchott K, Walther W, Bergmann S, Schla PM and Royer HD: Hyperthermia-induced nuclear translocation of transcription factor YB-1 leads to enhanced expression of multidrug resistance-related ABC transporters. J Biol Chem 276: 28562-28569, 2001.

13. Steiner E, Holzmann K, Pirker C, Elbling L, Micksche M, Sutterlüty $\mathrm{H}$ and Berger $\mathrm{W}$ : The major vault protein is responsive to and interferes with interferon- $\gamma$-mediated STAT1 signals. J Cell Sci 119: 459-469, 2006.

14. Steiner E, Holzmann K, Pirker C, Elbling L, Micksche M and Berger W: SP-transcription factors are involved in basal MVP promoter activity and its stimulation by HDAC inhibitors. Biochem Biophys Res Commun 317: 235-243, 2004.

15. Sawadogo M, Van Dyke MW, Gregor PD and Roeder RG: Multiple forms of the human gene-specific transcription factor USF. I. Complete purification and identification of USF from HeLa cell nuclei. J Biol Chem 263: 11985-11993, 1988.

16. Sawadogo M: Multiple forms of the human gene-specific transcription factor USF. II. DNA binding properties and transcriptional activity of the purified HeLa USF. J Biol Chem 263: 11994-12001, 1988.

17. Gregor PD, Sawadogo M and Roeder RG: The adenovirus major late transcription factor USF is a member of the helix-loop-helix group of regulatory proteins and binds to DNA as a dimer. Genes Dev 4: 1730-1740, 1990

18. Sirito M, Walker S, Lin Q, Kozlowski MT, Klein WH and Sawadogo M: Members of the USF family of helix-loop-helix proteins bind DNA as homo- as well as heterodimers. Gene Expr 2: 231-240, 1992.

19. Miyamoto NG, Moncollin V, Wintzerith M, Hen R, Egly JM and Chambon P: Stimulation of in vitro transcription by the upstream element of the adenovirus-2 major late promoter involves a specific factor. Nucleic Acids Res 12: 8779-8799, 1984.

20. Yu YT and Manley JL: Generation and functional analyses for base substitution mutants of the adenovirus 2 major late promoter. Nucleic Acids Res 12: 9309-9321, 1984.

21. Sirito M, Lin Q, Maity T and Sawadogo M: Ubiquitous expression of the 43- and 44-kDa forms of transcription factor USF in mammalian cells. Nucleic Acids Res 22: 427-433, 1994.

22. Shimamoto Y, Sumizawa T, Haraguchi M, Gotanda T, Jueng HC, Furukawa T, Sakata R and Akiyama S: Direct activation of the human major vault protein gene by DNA-damaging agents. Oncol Rep 15: 645-652, 2006. 